\title{
FINDING TWO VERTEX-DISJOINT CYCLES IN A GRAPH
}

\author{
THINH D. NGUYEN
}

\begin{abstract}
Given a graph $G(V, E)$, finding two vertex-disjoint cycles in it is a difficult problem. This paper shows this claim holds true.
\end{abstract}

\section{INTRODUCTION AND NOTATION}

Two cycles in a graph are vertex-disjoint if they have no common vertex. The maximum degree of a graph $G$ is the largest value of the degree of any vertex in it. This parameter of a graph $G$ is denoted by $\Delta(G)$. A graph in which every vertex has degree 3 is called a cubic graph. So, we want to show that, given a cubic graph $G(V, E)$, decide whether it contains two vertex-disjoint cycles of the same cardinality $|V| / 2$ is hard. As we shall see, the problem remains hard even if the input graph is further restricted to be planar cubic. We denote these two related problems by Balanced Two Cycles $\Delta=4$ and Balanced Two Cycles Cubic PLANAR

Claim 1.1. We have that $\mathrm{HC} \leq_{p}$ Balanced Two Cycles $\Delta=4$

Claim 1.2. We have that $\mathrm{HC} \leq_{p}$ Balanced Two Cycles Cubic PlanaR

In both of the claims, $\mathrm{HC}$ denotes the well-known Hamiltonian cycle. Garey and Johnson [1] proves its NP-hardness. Also, the definition of 2-factor of a graph is needed. Simply speaking, a 2-factor of a connected graph is two connected subgraphs of it. These two factors are separated by deleting some edges of the original graph.

\section{Details of the COnstruction}

2.1. Proof of the claim 1.1. Equivalently, the problem stated in the previous section 1 can be stated as finding a 2-factor of a graph where each factor contains a cycle visiting every vertex in it. This also means that both of the two factors are Hamiltonian. Also, each such factor has exactly $|V| / 2$ vertices.

Proof. (of claim 1.1) We proceed in two stages. The first one reduce Hamiltonian cycle in cubic graphs to Hamiltonian cycle going through a specified edge $r$ in a graph with $\Delta=4$. The second one reduce this later problem denoted $\mathrm{HC}^{\prime}$ below to Balanced Two Cycles $\Delta=4$, completing the proof of the first claim.

(1) It is known that $\mathrm{HC}$ in cubic graph is still $\mathbf{N P}$-complete.

(2) The following problem $\mathrm{HC}^{\prime}$ is also NP-complete:

Given a cubic graph $G$ and an edge $e$ in $G$, is there a Hamiltonian cycle

Key words and phrases. bridge, cycle, decomposition, graph, Hamiltonian.

Perebor. 
through $e$. Although this result has been present in literature. For selfcontainedness, we present a rather simple proof here.

We reduce from $\mathrm{HC}$ for cubic graph. The reduction is simple. For each vertex (in the left-hand side of the figure below), we replace it by a triangle with another vertex in it (as in the right-hand side of the same figure).
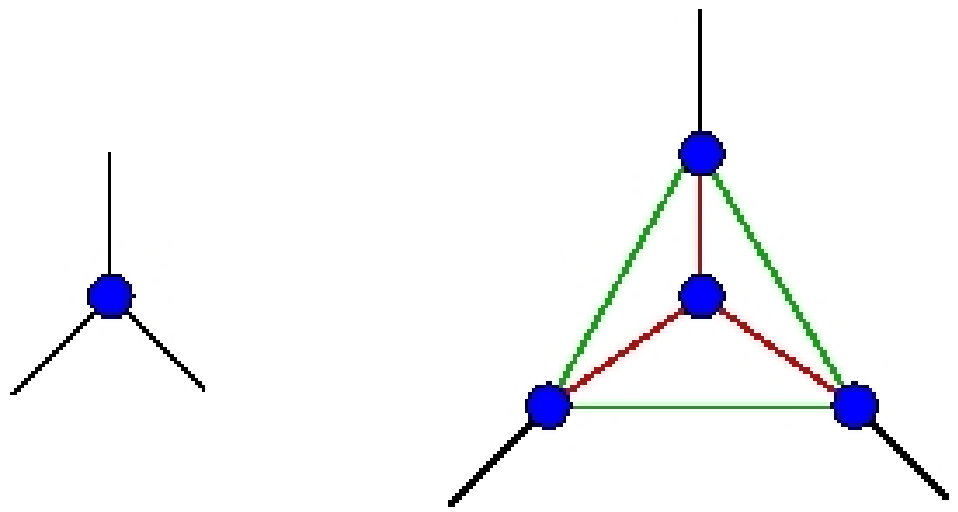

Fig. 1: Construction for $\Delta=4$

Call this new graph $G^{\prime}$. $G^{\prime}$ is Hamiltonian iff. $G$ is. Furthermore, if $G^{\prime}$ is Hamiltonian, then for any red edge, there is a Hamiltonian cycle that contains this red edge. Obviously, without the vertex in the middle, every Hamiltonian cycle contains two green edges and any green edge can then be replaced by two red ones. The reduction from $\mathrm{HC}$ to $\mathrm{HC}^{\prime}$ maps cubic graph $G$ to $G^{\prime}$ together with any red edge $e$. Note that $\Delta\left(G^{\prime}\right)=4$.

(3) Finally, we reduce $\mathrm{HC}^{\prime}$ to our first problem. Given an instance $(G, e)$, we create two copies of $G$, subdivide $e$ in each copy and connected the two vertices subdividing the two copies by a new edge $f$. This edge $f$ is a bridge in the new graph. If $G$ has a Hamiltonian cycle through $e$, then the new graph has a 2-factor consisting of two cycles of the same size. Conversely, if the new graph has such a 2 -factor, then it cannot use $f$. Therefore, it splits into two Hamiltonian cycles of the two copies. Since in these copies, the vertex subdividing $e$ has degree two, we get a Hamiltonian cycle in $G$ that contains $e$.

The above two reductions are two consecutive parts of our reduction and yields claim 1.1.

2.2. Proof of the claim 1.2. The above produced graphs only have bounded maximum degree. They lack bipartiteness, due to the triangles in the construction. We will give a small tweak to the previous proof as the proof in this subsection. This will maintain biartiteness and planarity simultaneously.

Proof. (of claim 1.2) We only need to change the gadget graph in item 2 above. By using this one, we avoid creating the triangles, so maintaining bipartiteness. 


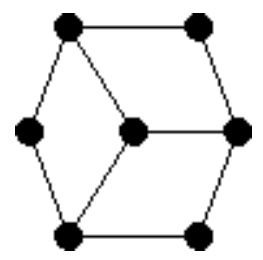

Fig. 2: Construction for bipartite planarity

The three vertices of degree 2 in that gadget will play the role of the vertices of the triangle above. The obtained graph has no odd cycle, is planar, and preserves cubicity. This yields the claim 1.2 .

\section{CONCLUSION}

As long as we study a mathematical conjecture, we should encourage ourselves of having enough labouring hours on Prasolov and Sharygin maths books. Then, reading some articles on theory of computing like [2] is a good practice. Only after that, could we think of the ultimate final for all mathematics sciences.

\section{REFERENCES}

1. Michael R. Garey, David S. Johnson, Computers and Intractability: A Guide to the Theory of NP-Completeness

2. Phan Dinh Dieu, Le Cong Thanh, Le Tuan Hoa, Average Polyno-mial Time Complexity of Some NP-Complete Problems, Theor. Comput. Sci. 46(3): 219-237 (1986)

Current address: Department of Mathematics, Moscow State University

Email address: kosmofarmer@yandex.com 